\title{
Patterns of Flavour Violation in the RSc Model, the LHT Model and Supersymmetric Flavour Models
}

\author{
Andrzej J. Buras* \\ Technical University Munich, Physics Department, D-85748 Garching, Germany, \\ TUM Institute for Advanced Study, D-80333 München, Germany \\ E-mail: aburas@ph.tum.de
}

\begin{abstract}
We summarize the results on patterns of flavour violation in a Randall-Sundrum model with custodial protection (RSc) and compare them with those identified in the Littlest Higgs Model with T-parity (LHT) and in a number of SUSY Flavour Models. While $K$ decays play in this presentation a prominent role, the inclusion of $B$ physics and lepton flavour violation is crucial in the distinction between these three popular extensions of the Standard Model (SM) by means of flavour physics.
\end{abstract}

2009 KAON International Conference KAONO9,

June 09 - 122009

Tsukuba, Japan

${ }^{*}$ Speaker. 


\section{Introduction}

Flavour changing neutral current processes (FCNC) will without any doubt provide a clear distinction between various new physics (NP) scenarios beyond the SM once the data on these processes will improve in the coming decade. The goal of this writing is to illustrate this fact by summarising patterns of flavour violation identified in 2006-2009 in my group at the TUM through intensive studies of a Randall-Sundrum model with custodial protection (RSc) [1, 2, 3, 4], the Littlest Higgs Model with T-Parity (LHT) [5, 6, 7, 8, 9, 10, 11, 12] and of a number of SUSY flavour models (SF) [13]. These three prominent directions beyond the SM contain new sources of $\mathrm{CP}$ and flavour violation implying thereby in certain cases spectacular deviations from the SM expectations and more generally from the patterns of flavour violation characteristic for models with minimal flavour violation (MFV).

In Section 2 we will briefly describe the RSc scenario and the results for FCNC processes obtained by us. The presentation of the LHT model in Section 3 is very short as an update of our efforts in this model appeared recently [12]. The same statement applies to SF models discussed in Section 4 with a very detailed analysis of these models presented in [13]. A brief comparison of these three NP scenarios and an outlook in Section 5 close this mini-review. Due to space limitations the list of references is incomplete and I apologize for it already here. I will improve on it in my EPS09 talk.

\section{FCNC Processes in the RSc Model}

\subsection{Express Review of the RS Framework [14]}

The story takes place in a $5 \mathrm{D}$ spacetime, where the extra dimension is compactified to the interval $0 \leq y \leq L$ with a warped metric given by

$$
d s^{2}=e^{-2 k y} \eta_{\mu v} d x^{\mu} d x^{v}-d y^{2}
$$

and $\eta_{\mu \nu}$ being the usual 4D Minkowski metric. There are two branes, the UV brane $(y=0)$ and the IR brane $(y=L)$ and the bulk between them. Energy scales are suppressed by the warp factor $\exp (-k y)$ providing a natural solution to the gauge hierarchy problem or equivalently to the vast disparity of the Planck and electroweak scales [14].

In the set up considered by us

1. Fermions (quarks and leptons) and gauge bosons, to be specified later on, live in the bulk $[15,16,17]$,

2. the Higgs boson is localised on the IR brane,

3. Kaluza-Klein (KK) excitations of fermions and gauge bosons live close to the IR brane,

4. the SM fermion (zero mode) shape functions depend exponentially on the bulk mass parameters $c_{i}$ that are characteristic for a given fermion

$$
f^{(0)} \sim e^{\left(1 / 2-c_{i}\right) k y} .
$$

Consequently fermions with $c_{i}>1 / 2$ are localized towards the UV brane, while the ones with $c_{i}<1 / 2$ towards the IR brane. As the Higgs lives on the IR brane, fermions with $c_{i}>1 / 2$ have only 
a small overlap with the Higgs and aquire masses much smaller than the Higgs vacuum expectation value (these are basically all SM fermions except the top quark). On the other hand for $c_{t}<1 / 2$ the large top quark mass is generated. Strictly speaking bulk parameters and shape functions for left- and right-handed fermions are involved and the elements of 4D Yukawa matrices are given in terms of the elements of the 5D Yukawa matrices as follows:

$$
\left(Y_{u, d}^{4 D}\right)_{i j}=\left(Y_{u, d}^{5 D}\right)_{i j} f_{i}^{Q} f_{j}^{u, d}
$$

with $\left(Y_{u, d}^{5 D}\right)_{i j}$ supposed to be anarchic. $f_{i}^{Q}$ and $f_{j}^{u, d}$ are the shape functions of left-handed and righthanded fermions, respectively, evaluated at the IR brane, where the Higgs is placed. This set up allows to generate the hierarchical structure of quark masses and CKM parameters in a natural manner with $\left(Y_{u, d}^{5 D}\right)_{i j}$ and $c_{i}$ being of order unity [17, 15]. The latter are typically in the range $0.3 \leq c_{i} \leq 0.6$

5. The shape functions of gluons and photons are flat due to exact $S U(3) \times U(1)_{Q}$ invariance. On the other hand the corresponding profiles of the $W^{ \pm}$and $Z^{0}$ gauge bosons, while being flat before the electroweak symmetry breaking (EWSB), are distorted by $v^{2} / M_{K K}^{2}$ corrections near the IR brane after EWSB took place. $M_{K K}$ is the KK scale. The 4D picture of this happening is the mixing of the heavy KK gauge bosons with the $W^{ \pm}$and $Z^{0}$ in the process of EWSB modifying thereby the couplings of the final light gauge boson mass eigenstates that are interpreted as the SM $W^{ \pm}$and $Z^{0}$.

6. Finally, the interactions between fermions and gauge bosons are given by the overlaps of the corresponding shape functions.

\subsection{First Phenomenological Implications}

This general set up with the SM gauge symmetry in the bulk has the following phenomenological implications:

A. The fact that fermions are localized at different positions in the bulk and the shape functions of $Z$ and $W^{ \pm}$gauge bosons are distorted near the IR brane after EWSB and the KK gauge bosons are peaked toward the IR brane implies non-universalities (in flavour) in the gauge interactions of fermions and gauge bosons. After the transformation to fermionic mass eigenstates FCNC transitions mediated by $Z$ and the heavy neutral KK gauge bosons including KK gluons take place at tree level. Thus the ordinary GIM mechanism is broken already at the tree level as opposed to the SM where it is broken first at the one-loop level. Fortunately, the gauge-fermion interactions in the RS framework exhibit also hierarchies and consequently a natural suppression of FCNC processes takes place. This suppression of FCNC transitions is known under the name of the RS-GIM [18, 19]. It saves the RS framework from a total disaster but as we will see below it is insufficient in the case of certain observables to provide appropriate suppression of FCNC transitions in the presence of the KK scales in the reach of the LHC.

B. The presence of FCNC transitions at the tree level is accompanied by the breakdown of the unitarity of the CKM matrix. Physically this breakdown originates from the mixing of the SM gauge bosons with the heavy KK gauge bosons on the one hand and from the mixing of the SM fermions with the KK fermions on the other hand.

C. The FCNC tree level exchanges of KK gluons imply the presence of the operators with the left-right (LR) Dirac structure that are very strongly suppressed in the SM and usually neglected. In 
the case of $\Delta F=2$ transitions, that is particle-antiparticle mixings, the Wilson coefficients of these operators are strongly enhanced through renormalization group effects and moreover in the case of $K^{0}-\bar{K}^{0}$ mixing their hadronic matrix elements are chirally enhanced. Together these effects amount relative to the contribution of the SM LL operator to an enhancement by a factor of 140 at the amplitude level if the KK scale at which this operator is generated is in the ballpark of $3 \mathrm{TeV}$.

D. The presence of three $3 \times 3$ hermitian bulk matrices $c^{Q}, c^{u}$ and $c^{d}$ in addition to the usual Yukawa couplings implies 27 new flavour and CP-violating parameters: 18 real mixing angles and 9 complex phases [19]. Thus the RS framework goes far beyond MFV.

E. Finally, the modifications in the electroweak gauge sector imply KK scales $M_{K K} \geq 2 \mathrm{TeV}$ and $M_{K K} \geq 10 \mathrm{TeV}$ in order to be consistent with the bounds on the $S$ and the $T$ parameter, respectively. Also NP contributions to the $Z b_{L} \bar{b}_{L}$ coupling become problematic.

The problems with electroweak parameters just mentioned lead several authors [20, 21, 22] to invent protection mechanisms that as we will see turned out to be useful also for suppressing FCNC processes. We will now discuss one particular model of this type.

\subsection{A RS Model with Custodial Protection (RSc)}

A more realistic class of RS models with the lowest KK excitations in the reach of the LHC has the following general structure. The bulk gauge symmetry is extended in order to obtain the usual custodial $S U(2)$ symmetry on the IR brane [20,21]. Additionally, in order to avoid problems with large NP contributions to the $Z b_{L} \bar{b}_{L}$ coupling, fermions are put into $S U(2)_{L} \leftrightarrow S U(2)_{R}$ symmetric representations [22]. Thus the bulk symmetry group is now

$$
G_{\text {bulk }}=S U(3)_{c} \times S U(2)_{L} \times S U(2)_{R} \times U(1)_{X} \times P_{L R}
$$

with $P_{L R}$ denoting the discrete symmetry interchanging $L$ and $R . U(1)_{X}$ allows to assign the usual hypercharges to quarks and leptons. $S U(2)_{R} \times U(1)_{X} \times P_{L R}$ is broken by boundary conditions on the UV brane down to $U(1)_{Y}$ and $S U(2)_{L} \otimes S U(2)_{R}$ is broken on the IR brane down to the custodial $S U(2)_{V}$ when the neutral component of the Higgs develops a vacuum expectation value. This also breaks the SM gauge group $S U(2)_{L} \times U(1)_{Y}$ down to $U(1)_{Q}$.

In this new set up the $T$ parameter and the $Z b_{L} \bar{b}_{L}$ coupling are protected from receiving NP contributions at the tree level up to small $P_{L R}$ symmetry breaking effects due to the boundary conditions on the UV brane. It has also been pointed out in $[1,2,4]$ that this construction when extended to three quark generations allows to protect certain flavour violating couplings from receiving tree level contributions. These are $Z \bar{d}_{L}^{i} d_{L}^{j}$ and $Z \bar{u}_{R}^{i} u_{R}^{j}$. On the other hand $Z \bar{d}_{R}^{i} d_{R}^{j}, Z \bar{u}_{L}^{i} u_{L}^{j}$ and $W^{+} \bar{u}_{L}^{i} d_{L}^{j}$ remain unprotected.

These are the general properties of the class of models in question. To be more concrete, fermion representations under the symmetry group have to be chosen. The particular fermion assignement in the model worked out by us in [3] has been motivated by the analyses of [23, 24, $25,26]$. In particular the fermion $S O(4)$ representations considered by us can easily be embedded into complete $S O(5)$ multiplets used in $[24,25,26]$ in the context of models with gauge-Higgs unification. Thus the SM doublets in our model belong to the representations $(2,2)$ under $S U(2)_{L} \times$ $S U(2)_{R}, u_{R}^{i}$ to $(1,1)$ and $d_{R}^{i}$ to $(1,3)$. Because of the $P_{L R}$ symmetry also $(3,1)$ representations for each generation not containing any of the SM particles have to be added. The implication of this 
assignement is the presence of KK quarks with electric charges $\pm 5 / 3$ that could in principle be discovered at the LHC. The fermion content of this model is explicitly given in [3], where also a complete set of Feynman rules has been worked out.

As far as the gauge boson sector is concerned, in addition to the SM gauge bosons the lightest KK gauge bosons are the KK-gluons, KK-photon and the electroweak KK gauge bosons $W_{H}^{ \pm}, W^{\prime \pm}$, $Z_{H}$ and $Z^{\prime}$, all with masses $M_{K K}$ around $2-3 \mathrm{TeV}$.

\subsection{Patterns of Flavour Violation}

\subsubsection{Preliminaries}

The first rough estimates of NP contributions to $\Delta F=2$ processes in RS scenarios can be found in $[27,19]$. However, the first more sophisticated analysis of these processes has been performed by Csaki, Falkowski and Weiler (CFW) [28], who included in their analysis the contributions of the dangerous LR operators generated by the tree level KK gluon exchanges and used the model independent bounds on the corresponding Wilson coefficients from the UTfit collaboration [29].

Assuming the $Y^{5 D}$ couplings to be anarchic and $\mathscr{O}(1)$ and describing the hierarchy of quark masses and weak mixings solely by the fermion shape functions, that is by the geometry in the fifth dimension, CFW found that the data on $\varepsilon_{K}$ imply a lower bound on $M_{K K}$ to be roughly $20 \mathrm{TeV}$, totally out of the LHC reach.

In view of this situation a RS-TUM team [1] has been built with the goal to investigate the amount of fine tuning in $Y^{5 D}$ couplings necessary to achieve an agreement of the theory with the data on $\varepsilon_{K}$ for the lowest values $M_{K K} \approx(2-3) \mathrm{TeV}$ that are consistent with EWP tests. Three additional RS-TUM teams [3, 2, 4] were supposed to study the details of a particular RS model and subsequently perform a number of phenomenological analyses.

As already advertised before, the electroweak and flavour structure of the model, including Feynman rules, has been worked out in [3]. The phenomenological applications of these results have been made in three separate analyses that we will summarise briefly now.

\subsubsection{Particle-Antiparticle Mixing}

In view of the problems with $\varepsilon_{K}$ we have first attacked $\Delta F=2$ processes in [1]. The main advances made in this paper w.r.t previous analyses are

- Full renormalization group analysis of $K^{0}-\bar{K}^{0}, B_{d}^{0}-\bar{B}_{d}^{0}$ and $B_{s}^{0}-\bar{B}_{s}^{0}$ mixings at the NLO level by means of the master formulae for the Wilson coefficients of the full set of operators given in [30].

- The inclusion of the contributions from all SM gauge bosons and their lowest KK excitations. The previous analyses concentrated on KK gluon contributions.

- Simultaneous phenomenology of $\varepsilon_{K}, \Delta M_{K}, \Delta M_{s, d}, S_{\psi K_{S}}, A_{\mathrm{SL}}^{q}$ and $\Delta \Gamma_{q}$.

- Relation of the RSc flavour model to the Froggatt-Nielsen approach [31] that allowed to derive analytic formulae for masses and mixings. An independent analysis of this type can be found in [32]. This group provided also another look at $\varepsilon_{K}$ [33]. 
- Calculation of the amount of fine tuning of $Y^{5 D}$ couplings, using the measure of Barbieri and Giudice $\Delta_{B G}\left(\varepsilon_{K}\right)$ [34], necessary to satisfy the data on $\varepsilon_{K}$ with $M_{K K} \approx 2-3 \mathrm{TeV}$.

The main results of these efforts are:

1. Confirmation of the CFW analysis [28] for anarchic $Y^{5 D}$ couplings: purely geometrical description of the quark masses and mixings and the measured value of $\varepsilon_{K}$ require $M_{K K} \geq 20 \mathrm{TeV}$.

2. Identification of the regions of parameter space with only modest fine tuning of $Y^{5 D}$, for which electroweak precision constraints and all $\Delta F=2$ constraints, in particular coming from $\varepsilon_{K}$ and $\Delta M_{K}$, are satisfied and the quark masses and mixings correctly reproduced with $M_{K K} \approx$ $2-3 \mathrm{TeV}$.

3. The pattern of NP contributions to $\Delta F=2$ processes turns out to be as follows:

- $\varepsilon_{K}$ and $\Delta M_{K}$ are dominated by KK gluon tree level exchanges and the operator $Q_{2}^{\mathrm{LR}}$ and not the standard LL operator $Q_{1}^{\mathrm{VLL}}$, both given as follows

$$
Q_{2}^{\mathrm{LR}}=\left(\bar{s} P_{L} d\right)\left(\bar{s} P_{R} d\right), \quad Q_{1}^{V L L}=\left(\bar{s} \gamma_{\mu} P_{L} d\right)\left(\bar{s} \gamma_{\mu} P_{L} d\right),
$$

with $P_{R, L}=\left(1 \pm \gamma_{5}\right) / 2$.

- In the case of $\Delta M_{d, s}, S_{\psi K_{S}}$ and $S_{\psi \phi}$ and generally in the case of $\Delta B=2$ observables, the LL operator $Q_{1}^{\mathrm{VLL}}$ and $Q_{2}^{\mathrm{LR}}$ compete with each other and both $Z_{H}$ and $\mathrm{KK}$ gluon contributions have to be taken into account.

- Most interestingly the CP asymmetry in $B_{s}$ decays, $S_{\psi \phi}$, can reach values as high as the central value 0.8 in the data from CDF and D0 collaborations to be compared with its SM value 0.04 .

\subsubsection{Rare $K$ and B Decays}

For the allowed region of parameter space satisfying $\Delta F=2$ constraints and corresponding to a moderate fine tuning of $Y^{5 D}$ characterised by $\Delta_{B G}\left(\varepsilon_{K}\right) \leq 20$ we have [2]

- calculated the branching ratios for $K^{+} \rightarrow \pi^{+} v \bar{v}, K_{L} \rightarrow \pi^{0} v \bar{v}, K_{L} \rightarrow \pi^{0} l^{+} l^{-}, K_{L} \rightarrow \mu^{+} \mu^{-}$, $B_{s, d} \rightarrow \mu^{+} \mu^{-}, B \rightarrow X_{s, d} \nu \bar{v}$,

- investigated correlations between various $\Delta F=1$ and $\Delta F=2$ processes.

The main results of our analysis are given as follows:

1. The NP contributions to the processes listed above are dominated by tree level $Z$ exchanges, but as the left-handed couplings are protected, this dominance is governed by the right-handed $Z$ couplings that are not protected by the custodial symmetry.

2. The branching ratios for $K^{+} \rightarrow \pi^{+} v \bar{v}, K_{L} \rightarrow \pi^{0} v \bar{v}, K_{L} \rightarrow \pi^{0} l^{+} l^{-}$can be enhanced relative to the SM expectations up to factors of 1.6, 2.5 and 1.4, respectively, when only moderate fine tuning in $\varepsilon_{K}$ is required. Otherwise the enhancements can be larger. $\operatorname{Br}\left(K^{+} \rightarrow \pi^{+} v \bar{v}\right)$ and $\operatorname{Br}\left(K_{L} \rightarrow\right.$ $\left.\pi^{0} v \bar{v}\right)$ can be simultaneously enhanced but this is not necessary as the correlations between these two branching ratios is not evident in this model. On the other hand $\operatorname{Br}\left(K_{L} \rightarrow \pi^{0} v \bar{v}\right)$ and $\operatorname{Br}\left(K_{L} \rightarrow\right.$ $\left.\pi^{0} l^{+} l^{-}\right)(l=e, \mu)$ are strongly correlated and the enhancement of one of these three branching ratios implies the enhancement of the remaining two. 
3. A large enhancement of the short distance part of $B r\left(K_{L} \rightarrow \mu^{+} \mu^{-}\right)$is possible, up to a factor of 2-3, but not simultaneously with $\operatorname{Br}\left(K^{+} \rightarrow \pi^{+} \nu \bar{v}\right)$.

4. More importantly simultaneous large NP effects in $S_{\psi \phi}$ and $K \rightarrow \pi \nu \bar{v}$ channels are very unlikely.

5. The branching ratios for $B_{s, d} \rightarrow \mu^{+} \mu^{-}$and $B \rightarrow X_{s, d} v \bar{v}$ remain SM-like: the maximal enhancements of these branching ratios amount to $15 \%$.

6. The relations $[35,36]$ between various observables present in models with constrained minimal violation can be strongly violated.

\subsubsection{Impact of KK Fermions}

Until now our analysis did not include the contributions from KK fermions that primarly affect the SM gauge-fermion couplings through their mixing with the SM fermions in the process of electroweak symmetry breaking. In [4] using the effective Lagrangian approach and integrating out the KK fermions from the low energy theory we have derived general formulae for KK corrections to the SM couplings that involve quarks, $W^{ \pm}, Z$ and the neutral Higgs. Our formulae can be applied to corrections from any vector-like fermions. Although they have been derived in a different manner and have a different appearence, they turn out to be equivalent to the ones presented earlier by del Aguila et al. [37, 38, 39].

Using these formulae we have demostrated explicitly that the custodial protection of $Z \bar{d}_{L}^{i} d_{L}^{j}$ and $Z \bar{u}_{R}^{i} u_{R}^{j}$ couplings remains valid in the presence of mixing with $\mathrm{KK}$ fermions which is guaranteed by the $P_{L R}$ symmetric fermion representations.

Subsequently we have calculated the impact of KK fermions on the unprotected couplings $Z \bar{d}_{R}^{i} d_{R}^{j}, Z \bar{u}_{L}^{i} u_{L}^{j}, W^{+} \bar{u}_{L}^{i} d_{L}^{j}$ and in particular analysed the violation of the unitarity of the CKM matrix and the generation of the right-handed couplings of the $W^{ \pm}$bosons $W^{+} \bar{u}_{R}^{i} d_{R}^{j}$ due to KK mixing.

Comparing these effects with the ones coming from KK gauge bosons analysed earlier [1,2], we concluded that the latter effects are generally significantly larger so that the impact of KK fermions with masses $\mathscr{O}\left(M_{K K}\right)$ on our previous results is minor. In total, the corrections to the CKM parameters are very small except $V_{t b}$ and $V_{t s}$, where they can reach $1-2 \%$.

Finally, we have verified that the effective Lagrangian approach in the case at hand is rather accurate by diagonalizing numerically, in the full theory, the relevant $18 \times 18,12 \times 12$ and $18 \times 12$ mass matrices.

\subsubsection{Summary}

The RS framework at large involves 18 new real flavour parameters and 9 new CP-violating phases in the quark sector that reside in three $3 \times 3$ hermitian bulk matrices $c^{Q}, c^{u}$ and $c^{d}$. It could appear then at first sight that this framework is not predictive. Yet at least in the RSc model a very clear pattern of flavour violation emerges from our studies after $\varepsilon_{K}$ in this model has been made consistent with the data for $M_{K K}=2-3 \mathrm{TeV}$ with only moderate tuning of $Y^{5 D}$ :

A. $S_{\psi \phi}$ can be much larger than in the SM and as large as 0.8 in the ballpark of the central values found by $\mathrm{CDF}$ and $\mathrm{D} 0$ collaborations.

B. $\operatorname{Br}\left(K_{L} \rightarrow \pi^{0} v \bar{v}\right)$ and $\operatorname{Br}\left(K^{+} \rightarrow \pi^{+} v \bar{v}\right)$ can be enhanced up to factors 2.5 and 1.6, respectively. 
C. Rare B decays turn out to be SM-like. In particular $\operatorname{Br}\left(B_{s, d} \rightarrow \mu^{+} \mu^{-}\right)$can be enhanced by at most $15 \%$.

D. Simultaneous enhancements of $S_{\psi \phi}$ and of the $K \rightarrow \pi \nu \bar{v}$ branching ratios are rather unlikely.

This pattern implies that in the case of the confirmation of large values of $S_{\psi \phi}$ by future experiments significant deviations of $\operatorname{Br}\left(K_{L} \rightarrow \pi^{0} v \bar{v}\right)$ and $B r\left(K^{+} \rightarrow \pi^{+} v \bar{v}\right)$ from their SM values in this framework are very unlikely. On the other hand SM-like value of $S_{\psi \phi}$ will open the road for large enhancements of these branching ratios that could be tested by KOTO at J-Parc and NA62 at CERN, respectively. Reviews of our work on the RSc model appeared in [40, 41, 36, 42, 43].

Finally, let me just mention that large NP contributions in the RS framework that require some tunings of parameters in order to be in agreement with the experimental data have been found in $B r\left(B \rightarrow X_{s} \gamma\right)$ [44], $B r(\mu \rightarrow e \gamma)$ [45, 46, 47] and EDM's [19, 48], that are all dominated by dipole operators. Also the new contributions to $\varepsilon^{\prime} / \varepsilon$ can be large [49].

New theoretical ideas addressing the issue of large FCNC transitions in the RS framework and proposing new protection mechanism leading occasionally to MFV can be found in $[50,51,52,53$, $54,55]$.

\section{LHT News}

\subsection{Preliminaries}

Other popular extensions of the SM is the Littlest Higgs model without [56] and with T-parity $[57,58]$ in which the Higgs boson is protected by a new global symmetry, possibly originating in a new fermionic system with new strong interactions with the corresponding scale $\Lambda=\mathscr{O}(10) \mathrm{TeV}$. The SM Higgs boson is a pseudo-Goldstone boson of this symmetry. In order to make this model consistent with electroweak precision tests and simultaneously having the new particles of this model in the reach of the LHC, a discrete symmetry, T-parity, has been introduced [57, 58]. Under T-parity all SM particles are even. Among the new particles only a heavy $+2 / 3$ charged T quark belongs to the even sector. Its role is to cancel the quadratic divergence in the Higgs mass generated by the ordinary top quark. The even sector and also the model without T-parity do not go beyond MFV [59, 60].

More interesting from the point of view of FCNC processes is the T-odd sector. It contains three doublets of mirror quarks and three doublets of mirror leptons that communicate with the SM fermions by means of heavy $W_{H}^{ \pm}, Z_{H}^{0}$ and $A_{H}^{0}$ gauge bosons. These interactions are governed by new mixing matrices $V_{H d}$ and $V_{H l}$ for down-quarks and charged leptons, respectively. The corresponding matrices in the up $\left(V_{H u}\right)$ and neutrino $\left(V_{H V}\right)$ sectors are obtained by means of the relations $V_{H u}^{\dagger} V_{H d}=V_{C K M}, V_{H v}^{\dagger} V_{H l}=V_{P M N S}^{\dagger}[61,7]$.

The following properties distinguish primarily this model from the RS framework:

1. The model has a smaller number of new flavour parameters: 7 real parameters and $3 \mathrm{CP}-$ violating phases in addition to $9+1$ in the SM quark sector.

2. Only SM operators are relevant in this model so that the absence of dangerous LR operators allows to satisfy $\Delta F=2$ constraints basically without any significant fine tuning [12]. Moreover non-perturbative uncertainties in this model are smaller than in the RSc framework as no new hadronic matrix elements are involved. 
3. The NP scales are by a factor of 3-5 lower than in the case of the RSc model, so that new fermions and new gauge bosons with masses below $1 \mathrm{TeV}$ are easily in the reach of the LHC.

4. There are no tree level FCNC transitions mediating $\Delta S=2$ and $\Delta B=2$ transitions as well as rare $K$ and $B_{s, d}$ decays. In the LHT model in all these processes the GIM mechanism is broken first at the one-loop level.

In 2006-2009 several LHT-TUM teams performed extensive analyzes of FCNC processes in the quark and the lepton sector in the LHT model. Selected reviews of our work can be found in $[62,63,64,65]$. Earlier we have also analysed the Littlest Higgs model without T-parity (LH) $[59,60]$. This model has no custodial protection relevant for EWP observables and the NP scale is shifted to $2-3 \mathrm{TeV}$. These higher NP scales and the fact that the LH model is of MFV type, makes this model phenomenologically less interesting than the LHT model and we will not discuss it here.

\subsection{LHT Update}

One of the disturbing features of our 2006 results in the LHT model was the presence of an UV logarithmic divergence in the Z-penguin diagram that we interpreted as the sensitivity to the unknown UV completion of this model. Such an UV sensitivity should not really be surprising in models of this type, in which the high energy theory is not specified, and in fact has been identified first in the LH model [60].

However it turns out that in deriving vertices in the T-odd sector that contained right-handed mirror fermions and Z-boson we have missed some $\mathscr{O}\left(v^{2} / f^{2}\right)$ corrections identified in 2008 by Goto et al [66] and del Aguila et al [67] during their study of $K \rightarrow \pi \nu \bar{v}$ and lepton flavour violating processes, respectively. The inclusion of this new contribution cancels the divergences mentioned above making the LHT model much less sensitive to a possible UV completion of this model. While it has not been understood whether the disappearance of all UV divergences is connected with the presence of T-parity, this is clearly a good news.

Recently our LHT-Rescue-Team [12] repeated all our previous phenomenological analyses, this time adding the missed corrections and updating the input parameters. It should be emphasised that all our calculations not involving $\mathrm{Z}$ penguin diagrams like particle-antiparticle mixings, $b \rightarrow s \gamma$, $\mu \rightarrow e \gamma, \tau \rightarrow \mu \gamma$ and several other processes are not affected by these findings. On the other hand the NP effects in $K^{+} \rightarrow \pi^{+} v \bar{v}, K_{L} \rightarrow \pi^{0} v \bar{v}, K_{L} \rightarrow \pi^{0} l^{+} l^{-}, \mu \rightarrow 3 e, \tau \rightarrow 3 \mu$ and few other transitions are now smaller than reported by us previously, although they are still sizable.

The main results of our 2006-2009 efforts in the LHT model to be compared with the outcome of our efforts in the RSc model summarized at the end of Section 2 are as follows.

1. $S_{\psi \phi}$ can be much larger than its SM value but typically smaller than found in the RSc model: values above 0.3 are rather unlikely.

2. $\operatorname{Br}\left(K_{L} \rightarrow \pi^{0} v \bar{v}\right)$ and $\operatorname{Br}\left(K^{+} \rightarrow \pi^{+} v \bar{v}\right)$ can be enhanced up to factors of 3 and 2.5, respectively. Therefore they can reach visibly larger values than in the RSc model. The allowed points in the $\operatorname{Br}\left(K_{L} \rightarrow \pi^{0} v \bar{v}\right)$ vs $\operatorname{Br}\left(K^{+} \rightarrow \pi^{+} v \bar{v}\right)$ plot cluster around two branches. On one of them $\operatorname{Br}\left(K^{+} \rightarrow \pi^{+} v \bar{v}\right)$ can reach maximal values while $\operatorname{Br}\left(K_{L} \rightarrow \pi^{0} v \bar{v}\right)$ is SM-like. Here $\operatorname{Br}\left(K^{+} \rightarrow\right.$ $\left.\pi^{+} v \bar{v}\right)$ can easily reach the central experimental value of E949 Collaboration at Brookhaven [68]. On the other one $\operatorname{Br}\left(K_{L} \rightarrow \pi^{0} v \bar{v}\right)$ can reach maximal values but $\operatorname{Br}\left(K^{+} \rightarrow \pi^{+} v \bar{v}\right)$ can be enhanced by at most a factor of 1.4 and therefore not reaching the central experimental values. No such 
correlations are found in the case of the RSc model and moreover in this model the maximal enhancements are smaller: 2.5 and 1.6 for $\operatorname{Br}\left(K_{L} \rightarrow \pi^{0} v \bar{v}\right)$ and $\operatorname{Br}\left(K^{+} \rightarrow \pi^{+} v \bar{v}\right)$, respectively. The central experimental value for the latter branching ratio therefore cannot be reached in this model. Some insights in this different behaviour have recently been provided in [69].

3. Rare B-decays turn out to be SM-like but they can deviate by more from the SM expectations than it is the case for RSc. In particular $\operatorname{Br}\left(B_{s, d} \rightarrow \mu^{+} \mu^{-}\right)$can be enhanced by $30 \%$ and a significant part of this enhancement comes from the T-even sector.

4. Simultaneous enhancements of $S_{\psi \phi}$ and of $\operatorname{Br}(K \rightarrow \pi \nu \bar{v})$ is similarly to the RSc scenario rather unlikely but this feature is less pronounced than in the RSc model.

5. $\operatorname{Br}(\mu \rightarrow e \gamma)$ in the LHT model can reach the upper bound of $2 \cdot 10^{-11}$ from the MEGA collaboration and in fact some fine tuning of the parameters is required to satisfy this bound [8, 67, 12]: either the corresponding mixing matrix in the mirror lepton sector has to be at least as hierarchical as the CKM matrix and/or the masses of mirror leptons carrying the same electric charge must be quasi-degenerate. Therefore if the MEG collaboration does not find anything at the level of $10^{-13}$, significant fine tuning of the LHT parameters will be required in order to keep $\mu \rightarrow e \gamma$ under control. Similar comments apply to the RSc scenario.

6. It is not possible to distinguish the LHT model from the RSc and the supersymmetric models discussed in Section 4 on the basis of $\mu \rightarrow e \gamma$ alone. On the other hand as pointed out in [8] such a distinction can be made by measuring any of the ratios $\operatorname{Br}(\mu \rightarrow 3 e) / \operatorname{Br}(\mu \rightarrow e \gamma)$, $B r(\tau \rightarrow 3 \mu) / B r(\tau \rightarrow \mu \gamma)$, etc. In supersymmetric models all these decays are governed by dipole operators so that these ratios are $\mathscr{O}(\alpha)[70,71,72,73,74]$. In the LHT model the LFV decays with three leptons in the final state are not governed by dipole operators but by Z-penguins and box diagrams and the ratios in question turn out to be by at least one order of magnitude larger than in supersymmetric models.

7. Recently also CP violation in $D^{0}-\bar{D}^{0}$ mixing has been analysed in the LHT model [11]. Observable effects at a level well beyond anything possible with CKM dynamics have been identified. Comparisons with CP violation in $K$ and $B$ systems should offer an excellent test of this NP scenario and reveal the specific pattern of flavour and CP violation in the $D^{0}-\bar{D}^{0}$ system predicted by this model.

\section{Supersymmetric Flavour (SF) Models}

\subsection{Preliminaries}

The general MSSM framework with very many new flavour parameters in the soft sector is not predictive and is plagued by flavour and CP problems: FCNC processes and electric dipole moments are generically well above the experimental data and upper bounds, respectively. Moreover the MSSM framework addressing primarily the gauge hierarchy problem and the quadratic divergences in the Higgs mass does not provide automatically the hierarchical pattern of quark and lepton masses and of FCNC and CP violating interactions.

Much more interesting from this point of view are supersymmetric flavour models with flavour symmetries that allow for a simultaneous understanding of the flavour structures in the Yukawa couplings and in SUSY soft-breaking terms, adequately suppressing FCNC and CP violating phenomena and solving SUSY flavour and CP problems. 
Supersymmetric models with flavour symmetries can be divided into two broad classes depending on whether they are based on abelian or non-abelian flavour symmetries. Moreover, their phenomenological output crucially depends on whether the flavour and CP violations are governed by left-handed currents or there is an important new right-handed current component [13]. They can be considered as generalisations of the Froggatt-Nielsen mechanism for generating hierarchies in fermion masses and their interactions but are phenomenologically much more successful than the original Froggatt-Nielsen model [31]. There is a rich literature on supersymmetric models based on flavour symmetries and I do not have space to refer to all of them here. A rather complete list of references can be found in a very recent paper from my group [13] that I will briefly summarise now.

\subsection{Patterns of Flavour Violation in the SF Models}

Recently one of the SUSY-TUM teams [13] performed an extensive study of processes governed by $b \rightarrow s$ transitions in the SF models and of their correlations with processes governed by $b \rightarrow d$ transitions, $s \rightarrow d$ transitions, $D^{0}-\bar{D}^{0}$ oscillations, LFV decays, electric dipole moments and $(g-2)_{\mu}$. Both abelian and non-abelian flavour models have been considered as well as the flavour blind MSSM (FBMSSM) and the MSSM with MFV. It has been shown how the characteristic patterns of correlations among the considered flavour observables allow to distinguish between these different SUSY scenarios and also to distinguish them from RSc and LHT scenarios of NP.

Of particular importance in our study were the correlations between the CP asymmetry $S_{\psi \phi}$ and $B_{s} \rightarrow \mu^{+} \mu^{-}$, between the observed anomalies in $S_{\phi K_{s}}$ and $S_{\psi \phi}$, between $S_{\phi K_{s}}$ and $d_{e}$, between $S_{\psi \phi}$ and $(g-2)_{\mu}$ and also those involving LFV decays.

In the context of our study of the SF models we have analysed the following representative scenarios:

i) dominance of right-handed (RH) currents (abelian model by Agashe and Carone[75]),

ii) comparable left- and right-handed currents with CKM-like mixing angles represented by the special version (RVV2) of the non abelian $S U$ (3) model by Ross, Velasco and Vives [76] as discussed recently in [77] and the model by Antusch, King and Malinsky (AKM) [78],

iii) dominance of left-handed (LH) currents in non-abelian models [79].

In the choice of these three classes of flavour models, we were guided by our model independent analysis, that I cannot discuss here because of the lack of space. Indeed these three scenarios predicting quite different patterns of flavour violation should give a good representation of most SF models discussed in the literature. The distinct patterns of flavour violation found in each scenario have been illustrated with several plots that can be found in figures 11-14 of [13].

The main messages from our analysis of the models in question are as follows:

1. Supersymmetric models with RH currents (AC, RVV2, AKM) and those with exclusively LH currents can be globally distinguished by the values of the CP-asymmetries $S_{\psi \phi}$ and $S_{\phi K_{S}}$ with the following important result: none of the models considered by us can simultaneously explain the $S_{\psi \phi}$ and $S_{\phi K_{S}}$ anomalies observed in the data. In the models with RH currents, $S_{\psi \phi}$ can naturally be much larger than its SM value, while $S_{\phi K_{S}}$ remains either SM-like or its correlation with $S_{\psi \phi}$ is 
inconsistent with the data. On the contrary, in the models with LH currents only, $S_{\psi \phi}$ remains SMlike, while the $S_{\phi K_{S}}$ anomaly can be easily explained. Thus already future precise measurements of $S_{\psi \phi}$ and $S_{\phi K_{S}}$ will select one of these two classes of models, if any.

2. The desire to explain the $S_{\psi \phi}$ anomaly within the models with RH currents unambiguously implies, in the case of the AC and the AKM models, values of $\operatorname{Br}\left(B_{s} \rightarrow \mu^{+} \mu^{-}\right)$as high as several $10^{-8}$. In the RVV2 model such values are also possible but not necessarily implied by the large value of $S_{\psi \phi}$. However, in all these models large values of $S_{\psi \phi}$ imply automatically the solution to the $(g-2)_{\mu}$ anomaly. Moreover, the ratio $\operatorname{Br}\left(B_{d} \rightarrow \mu^{+} \mu^{-}\right) / \operatorname{Br}\left(B_{s} \rightarrow \mu^{+} \mu^{-}\right)$in the AC and RVV2 models is dominantly below its MFV prediction and can be much smaller than the latter. In the AKM model this ratio stays much closer to the MFV value of roughly $1 / 33[80,81]$ and can be smaller or larger than this value with equal probability. Still, values of $\operatorname{Br}\left(B_{d} \rightarrow \mu^{+} \mu^{-}\right)$as high as $1 \times 10^{-9}$ are possible in all these models.

3. In the RVV2 and the AKM models, a large value of $S_{\psi \phi}$ combined with the desire to explain the $(g-2)_{\mu}$ anomaly implies $B r(\mu \rightarrow e \gamma)$ in the reach of the MEG experiment. In the case of the RVV2 model, $d_{e} \geq 10^{-29}$ e.cm. is predicted, while in the AKM model is typically smaller. Moreover, in the case of the RVV2 model, $\operatorname{Br}(\tau \rightarrow \mu \gamma) \geq 10^{-9}$ is then in the reach of Super-B machines, while this is not the case in the AKM model.

4. Next, while the abelian AC model resolves the present UT tensions [82, 83, 84, 85] through the modification of the ratio $\Delta M_{d} / \Delta M_{s}$, the non-abelian flavour models RVV2 and AKM provide the solution through NP contributions to $\varepsilon_{K}$. Moreover, while the AC model predicts sizable NP contributions to $D^{0}-\bar{D}^{0}$ mixing, such contributions are tiny in the RVV2 and AKM models.

5. The hadronic EDMs represent very sensitive probes of SUSY flavour models with righthanded currents. In the AC model, large values for the neutron EDM might be easily generated by both the up- and strange-quark (C)EDM. In the former case, visible CPV effects in $D^{0}-\bar{D}^{0}$ mixing are also expected while in the latter case large CPV effects in the $B_{s}$ system are unavoidable. The RVV2 and AKM models predict values for the down-quark (C)EDM and, hence for the neutron EDM, above the $\approx 10^{-28} e \mathrm{~cm}$ level when a large $S_{\psi \phi}$ is generated. All the above models predict a large strange-quark (C)EDM, hence, a reliable knowledge of its contribution to the hadronic EDMs, by means of lattice QCD techniques, would be of the utmost importance to probe or to falsify flavour models embedded in a SUSY framework.

6. In the supersymmetric models with exclusively LH currents, the desire to explain the $S_{\phi K_{S}}$ anomaly implies automatically the solution to the $(g-2)_{\mu}$ anomaly and the direct CP asymmetry in $b \rightarrow s \gamma$ much larger than its SM value. This is in contrast to the models with RH currents where this asymmetry remains SM-like.

7. Interestingly, in the LH-current-models, the ratio $\operatorname{Br}\left(B_{d} \rightarrow \mu^{+} \mu^{-}\right) / \operatorname{Br}\left(B_{s} \rightarrow \mu^{+} \mu^{-}\right)$can not only deviate significantly from its MFV value of approximately $1 / 33$, but in contrast to the models with RH currents considered by us can also be much larger than the latter value. Consequently, $\operatorname{Br}\left(B_{d} \rightarrow \mu^{+} \mu^{-}\right)$as high as $(1-2) \times 10^{-9}$ is still possible while being consistent with the bounds on all other observables, in particular the one on $\operatorname{Br}\left(B_{s} \rightarrow \mu^{+} \mu^{-}\right)$. Also interesting correlations between $S_{\phi K_{S}}$ and CP asymmetries in $B \rightarrow K^{*} \ell^{+} \ell^{-}$are found.

8. Finally the branching ratios for $K \rightarrow \pi v \bar{v}$ decays in the supersymmetric models considered by us remain SM-like and can be distinguished from RSc and LHT models where they can be significantly enhanced. 


\section{Summary and Outlook}

In our search for a fundamental theory the understanding of the observed flavour and $\mathrm{CP}$ violating interactions and the explanation of the hierarchical fermion spectrum is a very important goal. In this mini-review we have addressed in some detail FCNC processes in the RSc model, briefly summarized TUM results for these processes in the LHT model and made a propaganda for our recent very detailed analysis of FCNC processes, electric dipole moments and $(g-2)_{\mu}$ in a number of supersymmetric flavour models.

The patterns of flavour and $\mathrm{CP}$ violation in NP scenarios considered by us are in spite of many parameters involved sufficiently distinct that they can be distinguished from each other through experiments in the coming decade. This assumes that significant departures from the SM expectations will be found in a number of observables in $K, B_{s, d}$ and $D$ systems and in particular in LFV decays and EDMs. Also the persistent $(g-2)_{\mu}$ anomaly will play an important role in these considerations.

As an overture to various future possibilities let us consider the following two scenarios which could turn out soon to be reality.

Scenario 1. Let us assume that $S_{\psi \phi}$ has been found to be $0.30 \pm 0.05$, well beyond anything achievable by the CKM dynamics, but naturally found in the RSc model, in two SF models with RH currents (AC,RVV2) and with some efforts in the AKM and the LHT model. The SF models with LH-currents only, having $S_{\psi \phi}$ SM-like, will be ruled out.

First, this is rather bad news for the $K \rightarrow \pi v \bar{v}$ experiments if any of these models turns out to be chosen by nature: $\operatorname{Br}\left(K_{L} \rightarrow \pi^{0} v \bar{v}\right)$ and $\operatorname{Br}\left(K^{+} \rightarrow \pi^{+} v \bar{v}\right)$ will be SM-like in all these models. In the RSc and LHT models this is a consequence of a large $S_{\psi \phi}$. In the SF models $K \rightarrow \pi \nu \bar{v}$ decays are SM-like as the relevant NP effects are strongly suppressed in these models in the process of solving the SUSY-FCNC problem. Yet, there are other supersymmetric models where these decays can be strongly enhanced. But this story is for another occassion.

Now the distinction between RSc, LHT, AC, RVV2 and AKM models will be partly made through the measurement of $\operatorname{Br}\left(B_{s} \rightarrow \mu^{+} \mu^{-}\right)$. If this branching ratio will turn out to be $\mathscr{O}\left(10^{-8}\right)$, RSc and LHT will be ruled out, while such an enhancement of $\operatorname{Br}\left(B_{s} \rightarrow \mu^{+} \mu^{-}\right)$is a prediction of the AC and AKM models. In the RVV2 model this is not a prediction but such high values can be accomodated in this model. The AC and AKM models can then be distinguished through the manner they address various tensions in the standard UT analyses. While the AC model would favour $\gamma>80^{\circ}$ and $\alpha<80^{\circ}$ without NP contributions to $\varepsilon_{K}$, the AKM model can solve the recent $\varepsilon_{K}$ anomaly [83,85] with NP contributions to $\varepsilon_{K}$, while keeping $\gamma \approx 70^{\circ}$ and $\alpha \approx 90^{\circ}$ as suggested by UTfits and CKMfitter. Thus precise measurements of the angle $\gamma$ at the LHCb could select one of these models. Another distinction could be made through CP violation in the $D^{0}-\bar{D}^{0}$ oscillations that is predicted to be sizable in the AC model but small in the AKM model [13].

Scenario 2. Let us next assume that $S_{\psi \phi}$ has been found to be $0.30 \pm 0.05$, as in the previous example, but $\operatorname{Br}\left(B_{s} \rightarrow \mu^{+} \mu^{-}\right)$turns out to be SM-like, that is in the ballpark of $4 \cdot 10^{-9}$. AC and AKM models are then ruled out but RSc and LHT models will be in a good shape as they both expect $\operatorname{Br}\left(B_{s} \rightarrow \mu^{+} \mu^{-}\right)$to be SM-like. Similarly the RVV2 model will survive as it can accomodate such low values of this branching ratio in the presence of a substantial $S_{\psi \phi}$. The distinction between these three models can then be made by means of other observables but the upper bound on the 
number of pages for this contribution set by the organizers of KAON 09 has already been badly violated and I will not discuss it here. Readers, who are are still reading this sentence, are asked to look into our analysis in [13], where a DNA-Flavour Test has been proposed. This test should give still a deeper insight into the patterns of flavour violation in various scenarios, in particular when considered simultaneously with various correlations present in concrete models. The interplay of these efforts with the direct searches for NP will be most exciting.

Let me then close this mini-review of our work at TUM with the following note. In spite of no spectacular discoveries of NP in flavour violating processes this year, it was a very interesting workshop, very well organized and kept in a very pleasent atmosphere. I am happy I could be a part of it. While thanking the organisers of KAON 09 for inviting me to give this talk, I would also like to thank the members of various NP-TUM teams for very fruitful collaborations without which this talk would not be possible. Special thanks go to Monika Blanke for critical comments on the manuscript.

This research was partially supported by the Deutsche Forschungsgemeinschaft (DFG) under contract BU 706/2-1, the DFG Cluster of Excellence 'Origin and Structure of the Universe' and by the German Bundesministerium für Bildung und Forschung under contract 05HT6WOA.

\section{References}

[1] M. Blanke, A. J. Buras, B. Duling, S. Gori, and A. Weiler, $\Delta F=2$ Observables and Fine-Tuning in a Warped Extra Dimension with Custodial Protection, JHEP 03 (2009) 001, [arXiv: 0809 . 1073].

[2] M. Blanke, A. J. Buras, B. Duling, K. Gemmler, and S. Gori, Rare K and B Decays in a Warped Extra Dimension with Custodial Protection, JHEP 03 (2009) 108, [arXiv : 0812 . 3803].

[3] M. E. Albrecht, M. Blanke, A. J. Buras, B. Duling, and K. Gemmler, Electroweak and Flavour Structure of a Warped Extra Dimension with Custodial Protection, arXiv:0903.2415.

[4] A. J. Buras, B. Duling, and S. Gori, The Impact of Kaluza-Klein Fermions on Standard Model Fermion Couplings in a RS Model with Custodial Protection, arXiv: 0905.2318.

[5] M. Blanke et. al., Particle antiparticle mixing, $\varepsilon_{K}, \Delta \Gamma_{q}, A_{\mathrm{SL}}^{q}, A_{\mathrm{CP}}\left(B_{d} \rightarrow \psi K_{S}\right), A_{\mathrm{CP}}\left(B_{s} \rightarrow \psi \phi\right)$ and $B \rightarrow X_{s, d} \gamma$ in the littlest Higgs model with T-parity, JHEP 12 (2006) 003, [hep-ph/ 0605214$]$.

[6] M. Blanke et. al., Rare and CP-violating $K$ and $B$ decays in the Littlest Higgs model with T-parity, JHEP 01 (2007) 066, [hep-ph / 0610298$].$

[7] M. Blanke et. al., Another Look at the Flavour Structure of the Littlest Higgs Model with T-Parity, Phys. Lett. B646 (2007) 253-257, [hep-ph / 0609284 ].

[8] M. Blanke, A. J. Buras, B. Duling, A. Poschenrieder, and C. Tarantino, Charged Lepton Flavour Violation and $(g-2)_{\mu}$ in the Littlest Higgs Model with T-Parity: a clear Distinction from Supersymmetry, JHEP 05 (2007) 013, [hep-ph / 0702136 ].

[9] M. Blanke, A. J. Buras, S. Recksiegel, C. Tarantino, and S. Uhlig, Correlations between $\varepsilon^{\prime} / \varepsilon$ and rare $K$ decays in the littlest Higgs model with T-parity, JHEP 06 (2007) 082, [arXiv: 0704 . 3329].

[10] M. Blanke, A. J. Buras, S. Recksiegel, C. Tarantino, and S. Uhlig, Littlest Higgs Model with T-Parity Confronting the New Data on $D^{0}-\bar{D}^{0}$ Mixing, Phys. Lett. B657 (2007) 81-86, [hep-ph / 0703254$]$.

[11] I. I. Bigi, M. Blanke, A. J. Buras, and S. Recksiegel, $C P$ Violation in $D^{0}-\bar{D}^{0}$ Oscillations: General Considerations and Applications to the Littlest Higgs Model with T-Parity, JHEP 07 (2009) 097, [arXiv:0904.1545]. 
[12] M. Blanke, A. J. Buras, B. Duling, S. Recksiegel, and C. Tarantino, FCNC Processes in the Littlest Higgs Model with T-Parity: a 2009 Look, arXiv:0906. 5454.

[13] W. Altmannshofer, A. J. Buras, S. Gori, P. Paradisi, and D. M. Straub, Anatomy and Phenomenology of FCNC and CPV Effects in SUSY Theories, arXiv:0909.1333.

[14] L. Randall and R. Sundrum, A large mass hierarchy from a small extra dimension, Phys. Rev. Lett. 83 (1999) 3370-3373, [hep-ph/9905221].

[15] T. Gherghetta and A. Pomarol, Bulk fields and supersymmetry in a slice of AdS, Nucl. Phys. B586 (2000) 141-162, [hep-ph/0003129].

[16] S. Chang, J. Hisano, H. Nakano, N. Okada, and M. Yamaguchi, Bulk standard model in the Randall-Sundrum background, Phys. Rev. D62 (2000) 084025, [hep-ph/99124 98].

[17] Y. Grossman and M. Neubert, Neutrino masses and mixings in non-factorizable geometry, Phys. Lett. B474 (2000) 361-371, [hep-ph/9912408].

[18] S. J. Huber, Flavor violation and warped geometry, Nucl. Phys. B666 (2003) 269-288, [hep-ph/0303183].

[19] K. Agashe, G. Perez, and A. Soni, Flavor structure of warped extra dimension models, Phys. Rev. D71 (2005) 016002, [hep-ph/0 408134 ].

[20] K. Agashe, A. Delgado, M. J. May, and R. Sundrum, RS1, custodial isospin and precision tests, JHEP 08 (2003) 050, [hep-ph/ 0308036$].$

[21] C. Csaki, C. Grojean, L. Pilo, and J. Terning, Towards a realistic model of Higgsless electroweak symmetry breaking, Phys. Rev. Lett. 92 (2004) 101802, [hep-ph/0308038].

[22] K. Agashe, R. Contino, L. Da Rold, and A. Pomarol, A custodial symmetry for Zb $\bar{b}$, Phys. Lett. B641 (2006) 62-66, [hep-ph/ 0605341$].$

[23] G. Cacciapaglia, C. Csaki, G. Marandella, and J. Terning, A New Custodian for a Realistic Higgsless Model, Phys. Rev. D75 (2007) 015003, [hep-ph/ 0607146$].$

[24] R. Contino, L. Da Rold, and A. Pomarol, Light custodians in natural composite Higgs models, Phys. Rev. D75 (2007) 055014, [hep-ph/ 0612048$].$

[25] M. S. Carena, E. Ponton, J. Santiago, and C. E. M. Wagner, Light Kaluza-Klein states in Randall-Sundrum models with custodial SU(2), Nucl. Phys. B759 (2006) 202-227, [hep-ph/0607106].

[26] M. S. Carena, E. Ponton, J. Santiago, and C. E. M. Wagner, Electroweak constraints on warped models with custodial symmetry, Phys. Rev. D76 (2007) 035006, [hep-ph/ 0701055$].$

[27] G. Burdman, Constraints on the bulk standard model in the Randall-Sundrum scenario, Phys. Rev. D66 (2002) 076003, [hep-ph / 0205329$].$

[28] C. Csaki, A. Falkowski, and A. Weiler, The Flavor of the Composite Pseudo-Goldstone Higgs, JHEP 09 (2008) 008, [arXiv: 0804 . 1954].

[29] UTfit Collaboration, M. Bona et. al., Model-independent constraints on $\Delta F=2$ operators and the scale of new physics, JHEP 03 (2008) 049, [arXiv: 0707 . 0636].

[30] A. J. Buras, S. Jager, and J. Urban, Master formulae for $\Delta F=2 N L O-Q C D$ factors in the standard model and beyond, Nucl. Phys. B605 (2001) 600-624, [hep-ph/ 0102316 ]. 
[31] C. D. Froggatt and H. B. Nielsen, Hierarchy of Quark Masses, Cabibbo Angles and CP Violation, Nucl. Phys. B147 (1979) 277.

[32] S. Casagrande, F. Goertz, U. Haisch, M. Neubert, and T. Pfoh, Flavor Physics in the Randall-Sundrum Model: I. Theoretical Setup and Electroweak Precision Tests, JHEP 10 (2008) 094, [arXiv:0807.4937].

[33] M. Bauer, S. Casagrande, L. Gruender, U. Haisch, and M. Neubert, Little Randall-Sundrum models: $\varepsilon_{K}$ strikes again, arXiv:0811.3678.

[34] R. Barbieri and G. F. Giudice, Upper Bounds on Supersymmetric Particle Masses, Nucl. Phys. B306 (1988) 63.

[35] A. J. Buras, Minimal flavor violation, Acta Phys. Polon. B34 (2003) 5615-5668, [hep-ph/0310208].

[36] A. J. Buras, Testing the CKM Picture of Flavour and CP Violation in Rare K and B Decays and Particle-Antiparticle Mixing, arXiv:0904.4917.

[37] F. del Aguila and J. Santiago, Universality limits on bulk fermions, Phys. Lett. B493 (2000) 175-181, [hep-ph/0008143].

[38] F. del Aguila, M. Perez-Victoria, and J. Santiago, Effective description of quark mixing, Phys. Lett. B492 (2000) 98-106, [hep-ph/0 007160$].$

[39] F. del Aguila, M. Perez-Victoria, and J. Santiago, Observable contributions of new exotic quarks to quark mixing, JHEP 09 (2000) 011, [hep-ph / 0007316 ].

[40] B. Duling, K and B meson mixing in warped extra dimensions with custodial protection, arXiv:0901.4599.

[41] S. Gori, Patterns of Flavour Violation in a Warped Extra Dimensional Model with Custodial Protection, arXiv:0901.4704.

[42] M. Blanke, K and B Physics in the Custodially Protected Randall-Sundrum Model, arXiv:0908.2716.

[43] B. Duling, Predictions for Flavour Observables in a RS Model with Custodial Symmetry, arXiv:0908.3099.

[44] K. Agashe, A. Azatov, and L. Zhu, Flavor Violation Tests of Warped/Composite SM in the Two-Site Approach, arXiv:0810.1016.

[45] K. Agashe, A. E. Blechman, and F. Petriello, Probing the Randall-Sundrum geometric origin of flavor with lepton flavor violation, Phys. Rev. D74 (2006) 053011, [hep-ph/ 0606021$].$

[46] S. Davidson, G. Isidori, and S. Uhlig, Solving the flavour problem with hierarchical fermion wave functions, Phys. Lett. B663 (2008) 73-79, [arXiv : 0711 . 3376].

[47] K. Agashe, Relaxing Constraints from Lepton Flavor Violation in 5D Flavorful Theories, arXiv:0902.2400.

[48] E. O. Iltan, The effects of lepton KK modes on the lepton electric dipole moments in the Randall Sundrum scenario, Eur. Phys. J. C54 (2008) 583-590, [arXiv: 0708 . 3765].

[49] O. Gedalia, G. Isidori, and G. Perez, Combining Direct \& Indirect Kaon CP Violation to Constrain the Warped KK Scale, arXiv:0905.3264.

[50] C. Csaki, A. Falkowski, and A. Weiler, A Simple Flavor Protection for RS, arXiv:0806.3757. 
[51] G. Cacciapaglia et. al., A GIM Mechanism from Extra Dimensions, JHEP 04 (2008) 006, [arXiv:0709.1714].

[52] C. Cheung, A. L. Fitzpatrick, and L. Randall, Sequestering CP Violation and GIM-Violation with Warped Extra Dimensions, JHEP 01 (2008) 069, [arXiv: 0711 . 4421].

[53] J. Santiago, Minimal Flavor Protection: A New Flavor Paradigm in Warped Models, JHEP 12 (2008) 046, [arXiv:0806.1230].

[54] C. Csaki and D. Curtin, A Flavor Protection for Warped Higgsless Models, arXiv: 0904.2137.

[55] C. Csaki, G. Perez, Z. Surujon, and A. Weiler, Flavor Alignment via Shining in RS, arXiv:0907.0474.

[56] N. Arkani-Hamed, A. G. Cohen, E. Katz, and A. E. Nelson, The littlest Higgs, JHEP 07 (2002) 034, [hep-ph/0206021].

[57] H.-C. Cheng and I. Low, TeV symmetry and the little hierarchy problem, JHEP 09 (2003) 051 , [hep-ph/0308199].

[58] H.-C. Cheng and I. Low, Little hierarchy, little Higgses, and a little symmetry, JHEP 08 (2004) 061, [hep-ph/ 0405243$]$.

[59] A. J. Buras, A. Poschenrieder, and S. Uhlig, Particle antiparticle mixing, epsilon $(K)$ and the unitarity triangle in the littlest Higgs model, Nucl. Phys. B716 (2005) 173-198, [hep-ph/ 0410309 ].

[60] A. J. Buras, A. Poschenrieder, S. Uhlig, and W. A. Bardeen, Rare K and B decays in the littlest Higgs model without T-parity, JHEP 11 (2006) 062, [hep-ph/ 0607189 ].

[61] J. Hubisz, S. J. Lee, and G. Paz, The flavor of a little Higgs with T-parity, JHEP 06 (2006) 041, [hep-ph/0512169].

[62] M. Blanke and A. J. Buras, A guide to flavour changing neutral currents in the littlest Higgs model with T-parity, Acta Phys. Polon. B38 (2007) 2923, [hep-ph / 0703117$].$

[63] A. J. Buras and C. Tarantino, Quark and lepton flavour physics in the littlest Higgs model with T-parity, hep-ph/0702202.

[64] B. Duling, Lepton Flavor Violation in the LHT - A Clear Distinction from Supersymmetry, arXiv:0709.4413.

[65] S. Recksiegel, Flavour Physics in the Littlest Higgs Model with T-Parity: Effects in the K, $B_{d, s}$ and D systems, arXiv:0908.3117.

[66] T. Goto, Y. Okada, and Y. Yamamoto, Ultraviolet divergences of flavor changing amplitudes in the littlest Higgs model with T-parity, Phys. Lett. B670 (2009) 378-382, [arXiv : 0809 . 4753].

[67] F. del Aguila, J. I. Illana, and M. D. Jenkins, Precise limits from lepton flavour violating processes on the Littlest Higgs model with T-parity, arXiv:0811.2891.

[68] E949 Collaboration, A. V. Artamonov et. al., New measurement of the $K^{+} \rightarrow \pi^{+} v \bar{v}$ branching ratio, arXiv:0808.2459.

[69] M. Blanke, Insights from the Interplay of $K \rightarrow \pi \nu \bar{v}$ and $\varepsilon_{K}$ on the New Physics Flavour Structure, arXiv:0904.2528.

[70] J. R. Ellis, J. Hisano, M. Raidal, and Y. Shimizu, A new parametrization of the seesaw mechanism and applications in supersymmetric models, Phys. Rev. D66 (2002) 115013, [hep-ph/0206110]. 
[71] E. Arganda and M. J. Herrero, Testing supersymmetry with lepton flavor violating tau and mu decays, Phys. Rev. D73 (2006) 055003, [hep-ph/ 0510405$].$

[72] A. Brignole and A. Rossi, Anatomy and phenomenology of mu tau lepton flavour violation in the MSSM, Nucl. Phys. B701 (2004) 3-53, [hep-ph/ 0404211$].$

[73] P. Paradisi, Higgs-mediated $\tau \rightarrow \mu$ and $\tau \rightarrow e$ transitions in II Higgs doublet model and supersymmetry, JHEP 02 (2006) 050, [hep-ph / 0508054 ].

[74] P. Paradisi, Higgs-mediated $e \rightarrow \mu$ transitions in II Higgs doublet model and supersymmetry, JHEP 08 (2006) 047, [hep-ph / 0601100 ].

[75] K. Agashe and C. D. Carone, Supersymmetric flavor models and the $B \rightarrow \phi K_{S}$ anomaly, Phys. Rev. D68 (2003) 035017, [hep-ph/0304229].

[76] G. G. Ross, L. Velasco-Sevilla, and O. Vives, Spontaneous CP violation and non-Abelian family symmetry in SUSY, Nucl. Phys. B692 (2004) 50-82, [hep-ph / 0401064 ].

[77] L. Calibbi et. al., FCNC and CP Violation Observables in a SU(3)-flavoured MSSM, arXiv:0907.4069.

[78] S. Antusch, S. F. King, and M. Malinsky, Solving the SUSY Flavour and CP Problems with SU(3) Family Symmetry, JHEP 06 (2008) 068, [arXiv : 0708 . 1282].

[79] L. J. Hall and H. Murayama, A Geometry of the generations, Phys. Rev. Lett. 75 (1995) 3985-3988, [hep-ph/9508296].

[80] A. J. Buras, Relations between $\Delta M_{s, d}$ and $B_{s, d} \rightarrow \mu \bar{\mu}$ in models with minimal flavour violation, Phys. Lett. B566 (2003) 115-119, [hep-ph/ 0303060$].$

[81] T. Hurth, G. Isidori, J. F. Kamenik, and F. Mescia, Constraints on New Physics in MFV models: a model-independent analysis of $\Delta F=1$ processes, Nucl. Phys. $\mathbf{B 8 0 8}$ (2009) 326-346, [arXiv:0807.5039].

[82] E. Lunghi and A. Soni, Possible Indications of New Physics in $B_{d}$-mixing and in $\sin (2 \beta)$ Determinations, Phys. Lett. B666 (2008) 162-165, [arXiv: 0803 . 4340].

[83] A. J. Buras and D. Guadagnoli, Correlations among new CP violating effects in $\Delta F=2$ observables, Phys. Rev. D78 (2008) 033005, [arXiv: 0805 . 3887].

[84] E. Lunghi and A. Soni, Hints for the scale of new CP-violating physics from B-CP anomalies, arXiv:0903.5059.

[85] A. J. Buras and D. Guadagnoli, On the consistency between the observed amount of CP violation in the $K$ - and $B_{d}$-systems within minimal flavor violation, arXiv:0901.2056. 